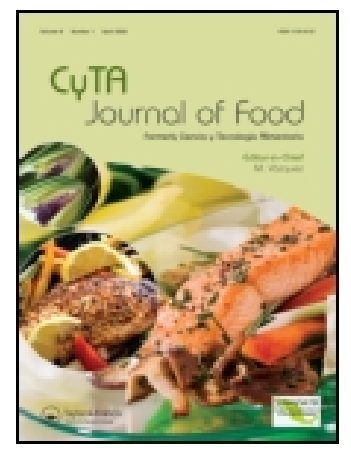

CYTA - Journal of Food

\title{
EVALUACIÓN PRELIMINAR DE LA PRESENCIA DE PESTICIDAS ORGANOCLORADOS EN PESCADOS DE LA PRESA VICENTE GUERRERO (TAMAULIP AS, MÉXICO) PRELIMINARY EVALUATION OF THE PRESENCE OF ORGANOCHLORINE PESTICIDES IN FISH OF VICENTE GUERRERO DAM (TAMAULIP AS, MEXICO)
}

R. M. Uresti-Marín, R. Santiago-Adame , N. E. Díaz-Moroles , J. GutiérrezLozano , M. Vázquez \& J. A. Ramírez de León

To cite this article: R. M. Uresti-Marín , R. Santiago-Adame , N. E. Díaz-Moroles , J. GutiérrezLozano , M. Vázquez \& J. A. Ramírez de León (2008) EVALUACIÓN PRELIMINAR DE LA PRESENCIA DE PESTICIDAS ORGANOCLORADOS EN PESCADOS DE LA PRESA VICENTE GUERRERO (TAMAULIP AS, MÉXICO) PRELIMINARY EVALUATION OF THE PRESENCE OF ORGANOCHLORINE PESTICIDES IN FISH OF VICENTE GUERRERO DAM (TAMAULIP AS, MEXICO), CYTA - Journal of Food, 6:1, 48-55, DOI: 10.1080/11358120809487627

To link to this article: https://doi.org/10.1080/11358120809487627

\section{(2) Copyright Taylor and Francis Group, LLC 曲 Published online: 02 Oct 2009.}

Submit your article to this journal 


\title{
EVALUACIÓN PRELIMINAR DE LA PRESENCIA DE PESTICIDAS ORGANOCLORADOS EN PESCADOS DE LA PRESA VICENTE GUERRERO (TAMAULIPAS, MÉXICO)
}

\section{PRELIMINARY EVALUATION OF THE PRESENCE OF ORGANOCHLORINE PESTICIDES INFISHOF VICENTE GUERRERODAM(TAMAULIPAS,MEXICO)}

\author{
Uresti-Marín, R. M. ${ }^{1 *}$; Santiago-Adame, R. ${ }^{1}$; Díaz-Moroles, N. E. ${ }^{1}$; Gutiérrez-Lozano, J. ${ }^{1}$; \\ Vázquez, M. ${ }^{2}$; Ramírez de León, J. A. ${ }^{1}$ \\ ${ }^{1}$ Departamento de Seguridad Alimentaria y del Medio Ambiente. UAM Reynosa-Aztlán. Universidad Autónoma de \\ Tamaulipas. Calle 16 y Lago de Chapala s/n. Col. Aztlán. C.P. 88740. Reynosa, Tamaulipas, México. \\ ${ }^{2}$ Departamento de Química Analítica, Nutrición y Bromatología. Facultad de Veterinaria. Universidad de Santiago de \\ Compostela, 27002 Lugo, España.
}

Recibido/Received 05-02-2008; aceptado/accepted 20-04-2008

*Autor para la correspondencia/Corresponding author. E-mail: ruresti@ uat.edu.mx

\begin{abstract}
Organochlorine pesticides (OCPs) are classified as persistent organic pollutants. The presence of OCPs in the environment is of global concern as they are persistent, ubiquitous and highly toxic. The purpose of this study was to evaluate the presence of organochlorine pesticides (aldrin, endrin, chlordane, mirex, heptachlor, DDT, DDE and DDD) in the muscle of four fish species from Vicente Guerrero Dam in Tamaulipas (Mexico): catfish (Ictalurus punctatus), carp (Ciprynus carpio), largemouth bass (Micropterus salmoides) and tilapia (Oreochromis niloticus). Solid phase extraction and gas chromatography with electron capture detector was used to detect the pesticides. The highest concentration found was for DDE in largemouth bass, carp and catfish $(239.90,165.98$ and $212.31 \mathrm{ng} / \mathrm{g}$, respectively) and for chlordane in catfish and largemouth bass (44.66 and $64.22 \mathrm{ng} / \mathrm{g}$ ). There is a risk of carcinogenic effects if carp and largemouth bass are regularly consumed by the population in the region as the concentration of $p-p$ '-DDE is higher than the recommendations of the US Environmental Protection Agency.
\end{abstract}

\section{Resumen}

Los pesticidas organoclorados (OCPs por sus siglas en inglés) están clasificados como contaminantes orgánicos persistentes. La presencia de OCPs en el ambiente es de interés mundial debido a que son persistentes, ubicuos y altamente tóxicos. El objetivo de este trabajo fue evaluar la presencia de pesticidas organoclorados (aldrín, endrín, clordano, mirex, heptacloro, DDT, DDE, DDD) en el músculo de 4 especies de pescado de la Presa Vicente Guerrero del estado de Tamaulipas (México): bagre (Ictalurus punctatus); carpa (Ciprynus carpio), lobina (Micropterus salmoides) y tilapia (Oreochromis niloticus). Para la determinación de los pesticidas se usó extracción en fase sólida y cromatografía de gases con detector de captura de electrones. La mayor concentración encontrada fue para el DDE en lobina, carpa y bagre (239,90, 165,98 y 212,31 $\mathrm{ng} / \mathrm{g}$, respectivamente) y para el clordano en bagre y lobina $(44,66$ y 64,22 ng/g). Existe riesgo de cáncer por el consumo de carpa y lobina en forma regular por parte de la población que habita en la región debido a que los altos niveles de $p$ - $p$ '-DDE encontrados superan las recomendaciones de la Agencia de Protección del Medio Ambiente de Estados Unidos.

Keywords: Organochlorine pesticides, fish muscle, gas chromatography, SPE, GC/ECD

Palabras clave: Pesticidas organoclorados, músculo de pescado, cromatografía de gases, SPE, GC/ECD

\section{INTRODUCCIÓN}

El Convenio de Estocolmo sobre contaminantes orgánicos persistentes (UNEP, 2001) incluye a los pesticidas organoclorados (OCPs). Estos pesticidas se bioacumulan en los tejidos lipídicos y se biomagnifican a través de la cadena alimenticia, lo cual implica un riesgo para la salud humana (Cruz et al., 2003). Además son ubicuos, persistentes y viajar grandes distancias (Iwata $e t$ al., 1994). Todas estas propiedades los hacen muy peligrosos (Pazos et al., 1988). El criterio utilizado por el
Convenio de Estocolmo establece que se consideran bioacumulables las sustancias que presenten un factor de bioacumulación (BAF) o de bioconcentración (BCF) en especies acuáticas mayor a 5000 o que presenten el log Kow mayor a 5 (UNEP, 2007). El Convenio de Estocolmo se centra en los contaminantes orgánicos persistentes (POPs) considerados más peligrosos y denominados «la docena sucia». De ellos, ocho son utilizados como plaguicidas: aldrín, clordano, DDT, dieldrín, endrín, heptacloro, mirex y toxafeno. Dos son productos químicos de aplicación industrial: bifenilos policlorados (más 
conocidos como PCBs) y hexaclorobenceno (que también es usado como plaguicida) y dos son subproductos no deseados: dioxinas y furanos. Los pesticidas organoclorados son considerados disruptores endocrinos (Amaral Mendes, 2002; Brody y Rudel, 2003; Calle et al., 2002; Vinggaard et al., 1999). La mayoría de los estudios para detectar concentraciones de riesgo en seres humanos se ha realizado principalmente en países altamente desarrollados (Lino y Silveira, 2006).

El gobierno de México es signatario del Convenio de Estocolmo, cuyo objetivo es proteger la salud humana $\mathrm{y}$ al ambiente de los POPs. A partir de la entrada en vigor de dicho instrumento, el 17 de mayo de 2004, existe el compromiso de elaborar un Plan Nacional de Implementación (PNI), para lo cual se requiere contar con elementos de diagnóstico tales como los niveles de POPs detectados en diferentes ambientes de la República Mexicana (www.ine.gob.mx).

En México, la lista de los plaguicidas autorizados y restringidos se encuentra en el Catálogo Oficial de Plaguicidas, donde además se indican los cultivos, los días de intervalo necesarios a respetar después de cada aplicación y los límites máximos de residuos autorizados. Este catálogo es publicado por la Comisión Intersecretarial para el Control y uso de Plaguicidas, Fertilizantes, y Sustancias Tóxicas (CICOPLAFEST), que incluye a la Secretaría de Agricultura, Ganadería, Desarrollo Rural, Pesca y Alimentación (SAGARPA), Secretaría del Medio Ambiente y Recursos Naturales (SEMARNAT), Secretaría de Salud (SS) y la Secretaría de Economía (SE).

Dentro de los principales plaguicidas con OCPs que están prohibidos desde 1982 se citan: aldrín, endrín y mirex, además del heptacloro (en 1992) y dieldrín (Diario Oficial de la Federación del 3 de enero de 1991). Entre los plaguicidas cuya comercialización y uso (pero no su producción) están prohibidos desde 1992 encontramos al hexaclorobenceno y al toxafeno.

El DDT se señala como plaguicida severamente restringido y su uso está limitado a campañas sanitarias en el combate a vectores de enfermedades transmisibles. El clordano se encuentran severamente restringidos para combatir termitas, que junto con los bifenilos policlorados (PCBs) y el mercurio forman parte del Programa de Gestión Ambiental de Sustancias Tóxicas de Atención Prioritaria a cargo del Instituto Nacional de Ecología (INE).

En México el clordano fue empleado en la década de 1970 para el combate de plagas del maíz y sorgo. Sin embargo, por su persistencia y toxicidad en 1988 se restringió únicamente para el control de termitas en áreas urbanas. El clordano nunca fue producido en México, sino importado de Estados Unidos en forma de producto técnico, a partir del cual se hacían las formulaciones para obtener los productos finales (INE, 2001).

En México existen estudios para detectar concentraciones de OCPs de riesgo para la salud, realizados en diferentes matrices como pescado (Gold-
Bouchot et al., 2006), agua (Hernández-Romero et al., 2004), sedimentos, agua y biota (Carvalho et al., 2002), músculo de pescado (Lewis et al., 2002; Favari et al., 2002), sedimentos (Botello et al., 2000; Lewis et al., 2000), algas, invertebrados y pescado (Lewis et al., 1998), camarón (Galindo-Reyes et al., 1996) y suelo, polvo y en sangre de niños expuestos a varios pesticidas (HerreraPortugal, 2005).

Tamaulipas es una región agrícola, ganadera y pesquera. La Presa Vicente Guerrero es un embalse artificial construido por el Gobierno Federal, con el propósito de captar agua procedente de los ríos Purificación, Corona y Pilón, para aprovecharla con fines de riego, abastecimiento a poblaciones humanas y control de avenidas. Posee una capacidad de 5283 millones de $\mathrm{m}^{3}$ con un vaso de $400 \mathrm{~km}^{2}$ que irriga 42000 hectáreas de terrenos agrícolas en Abasolo y Soto La Marina, esta presa desagua sus escurrimientos en el Golfo de México a la altura del poblado «La Pesca».

En esta presa las especies de pescado bagre, carpa y tilapia se capturan con fines comerciales para consumo humano. La lobina se utiliza en la pesca deportiva y es usada en forma limitada para consumo humano por los habitantes de la región. El tamaño permitido para la captura de la lobina en la Presa Vicente Guerrero es de 30 a 45 $\mathrm{cm}$. No deben capturarse especies de 45 a $55 \mathrm{~cm}$ y sólo se permiten ejemplares con tamaño superior a $55 \mathrm{~cm}$ en 10 s torneos deportivos autorizados. La población que vive en la zona de la presa puede capturar para consumo propio un máximo de $5 \mathrm{~kg}$ diarios de lobina del tamaño comprendido entre los 30 y $45 \mathrm{~cm}$.

El objetivo del presente trabajo fue evaluar la presencia de pesticidas organoclorados en bagre (Ictalurus punctatus), carpa (Ciprynus carpio), lobina (Micropterus salmoides) y tilapia (Oreochromis niloticus) de la Presa Vicente Guerrero en Tamaulipas, México.

\section{MATERIALES Y MÉTODOS}

Las muestras de las 4 especies de pescado (Ictalurus punctatus, Ciprynus carpio, Micropterus salmoides y Oreochromis niloticus) se adquirieron en una cooperativa pesquera local. Se capturaron durante el período de invierno de 2004 en la Presa Vicente Guerrero. Los especímenes analizados en este estudio fueron los peces más grandes del total de peces obtenidos en la pesca del día. Los pescados fueron eviscerados, fileteados y empacados en bolsas de polietileno debidamente etiquetados para su congelación a $-20^{\circ} \mathrm{C}$ hasta su análisis. Para su estudio, los filetes de pescado se descongelaron y molieron. Se determinó la humedad en las muestras de pescado por gravimetría. Para obtener el contenido de grasa en el músculo se utilizó un equipo soxhlet automatizado (Soxtec, HT2, Tecator, USA) utilizando el método 3541 aprobado por la EPA. Para la extracción de los OCPs en el 


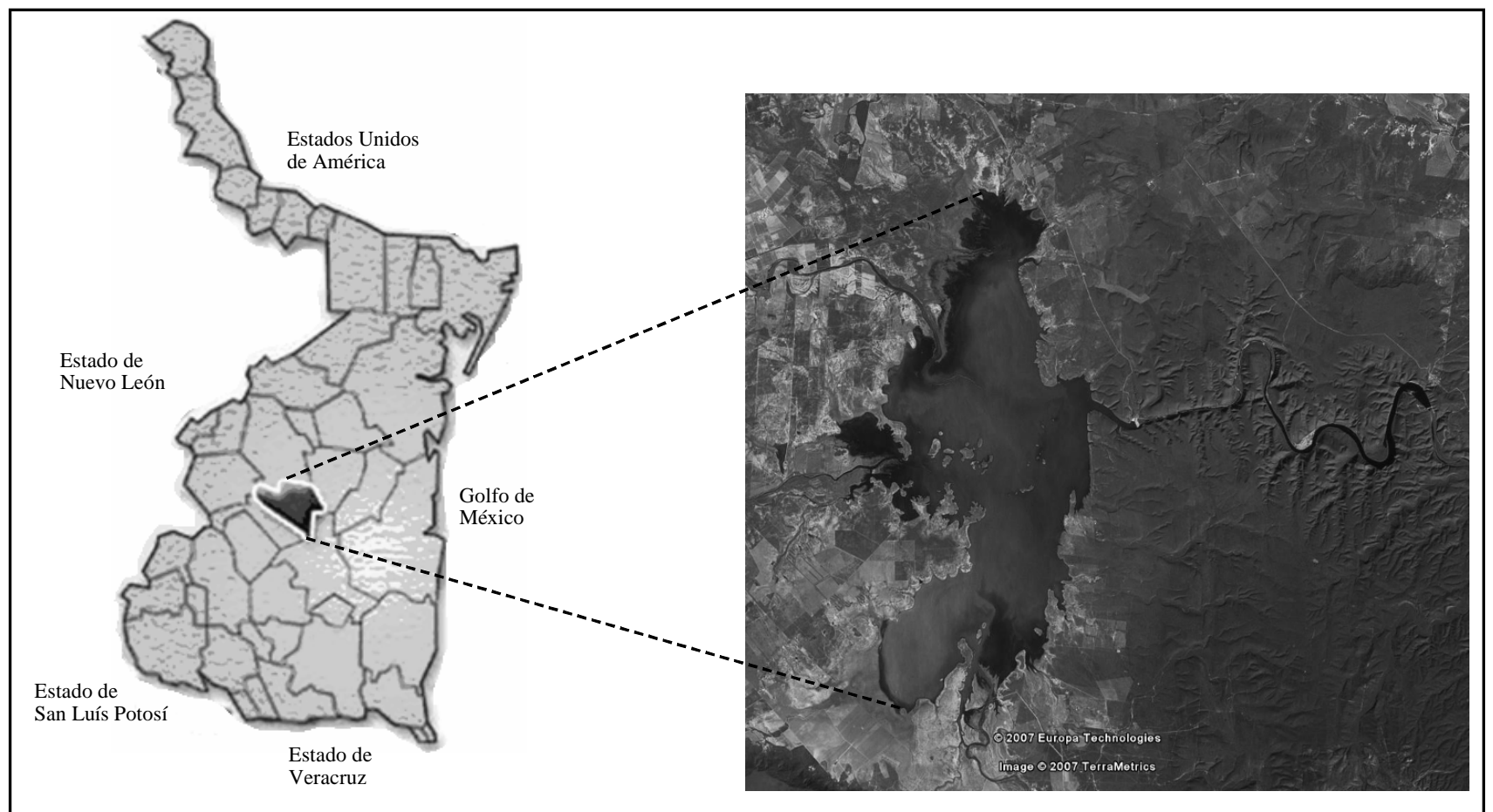

Figura 1. Ubicación de la Presa Vicente Guerrero en Tamaulipas, México. Fuente: google Earth.

Figure 1. Map showing location of the Vicente Guerrero Dam in Tamaulipas, Mexico. Source: Google Earth

músculo se usó la técnica de extracción en fase sólida (SPE) en columnas de octadecilo y de Florisil. Se seleccionó SPE debido a que es un procedimiento sencillo y de bajo costo que permite procesar varias muestras simultáneamente y es aplicable a diferentes analitos en matrices diversas. Se siguió la metodología de Schenck et al. (1994, 1996) con la modificación de usar una relación éter etílico: éter de petróleo de 10:90 v/v (en vez de 2:98 v/v) en la proporción de solventes utilizados para la elución. Otros autores han aplicado esta misma técnica para la determinación de OCPs en pescado y en grasa de res (Sun et al., 2000, 2003).

Para asegurar la limpieza del material de vidrio se siguió el procedimiento descrito en el método 1668 A de la EPA. Todos los solventes utilizados fueron grado HPLC o plaguicida: metanol, acetona, cloruro de metileno, éter etílico, tolueno, isooctano, éter de petróleo (TEDIA, USA). Los estándares de plaguicidas organoclorados utilizados fueron marca Chem Service con certificado de pureza: heptacloro, (99,1\%); aldrín, (98,6\%); endrín, (99\%); mirex, (99,4\%); 4.4' DDT, (98,6 \%); 4.4'-DDE, (99,5 $\%) ; 4.4^{\prime}$-DDD (99,3\%); $\gamma$-clordano y $\alpha$-clordano (mezcla de isómeros).

Se preparó una serie de 10 soluciones estándares individuales de pesticidas organoclorados. En balanza analítica se pesaron $0,01 \mathrm{~g}$ de cada pesticida en polvo y se aforó a $100 \mathrm{~mL}$ con isooctano para obtener una concentración de $100 \mathrm{mg} / \mathrm{L}$. A partir de estas soluciones se hicieron mezclas y diluciones para obtener las curvas de calibración.
Para la separación y cuantificación se utilizó un cromatógrafo de gases (GC) modelo HP 6890N (Agilent Technologies, USA) con un detector de captura de electrones $\left(\mu\right.$-ECD) ${ }^{63} \mathrm{Ni}$, equipado con automuestreador de la serie 7683, columna capilar Zebron ZB-5 (Phenomenex) de $30 \mathrm{~m} \mathrm{x} \mathrm{0,25} \mathrm{mm} \mathrm{D.} \mathrm{I.,} \mathrm{0,25} \mathrm{mm} \mathrm{de}$ espesor de película.

En el GC/ECD se usó el método 8081A de la Agencia de Protección Ambiental (EPA) de Estados Unidos. Las condiciones de operación fueron las siguientes: puerto de inyección en modo splitless, con volumen de inyección de $1 \mu \mathrm{L}$ y con el siguiente programa de temperatura: al inicio $70{ }^{\circ} \mathrm{C}$ durante 2 minutos, luego una rampa de $25{ }^{\circ} \mathrm{C} / \mathrm{min}$ hasta llegar a $150{ }^{\circ} \mathrm{C}$, después 3 ${ }^{\circ} \mathrm{C} / \mathrm{min}$ hasta llegar a $200{ }^{\circ} \mathrm{C}$, posteriormente $8{ }^{\circ} \mathrm{C} / \mathrm{min}$ hasta $280^{\circ} \mathrm{C}$ y mantener por $10 \mathrm{~min}$, finalmente se bajó la temperatura a $70{ }^{\circ} \mathrm{C}$ y se mantuvo por $3 \mathrm{~min}$. El detector se mantuvo en una temperatura de $320{ }^{\circ} \mathrm{C}$ y un flujo de nitrógeno de $60 \mathrm{~mL} / \mathrm{min}$.

Para evaluar las recuperaciones de los analitos se fortificaron muestras de músculo de pescado y se sometieron al procedimiento de SPE-GC/ECD.

\section{RESULTADOS Y DISCUSIÓN}

La Figura 1 presenta el mapa del estado de Tamaulipas y la ubicación de la Presa «Vicente Guerrero». Esta presa es la más grande del estado, se sitúa a $54 \mathrm{~km}$ al 


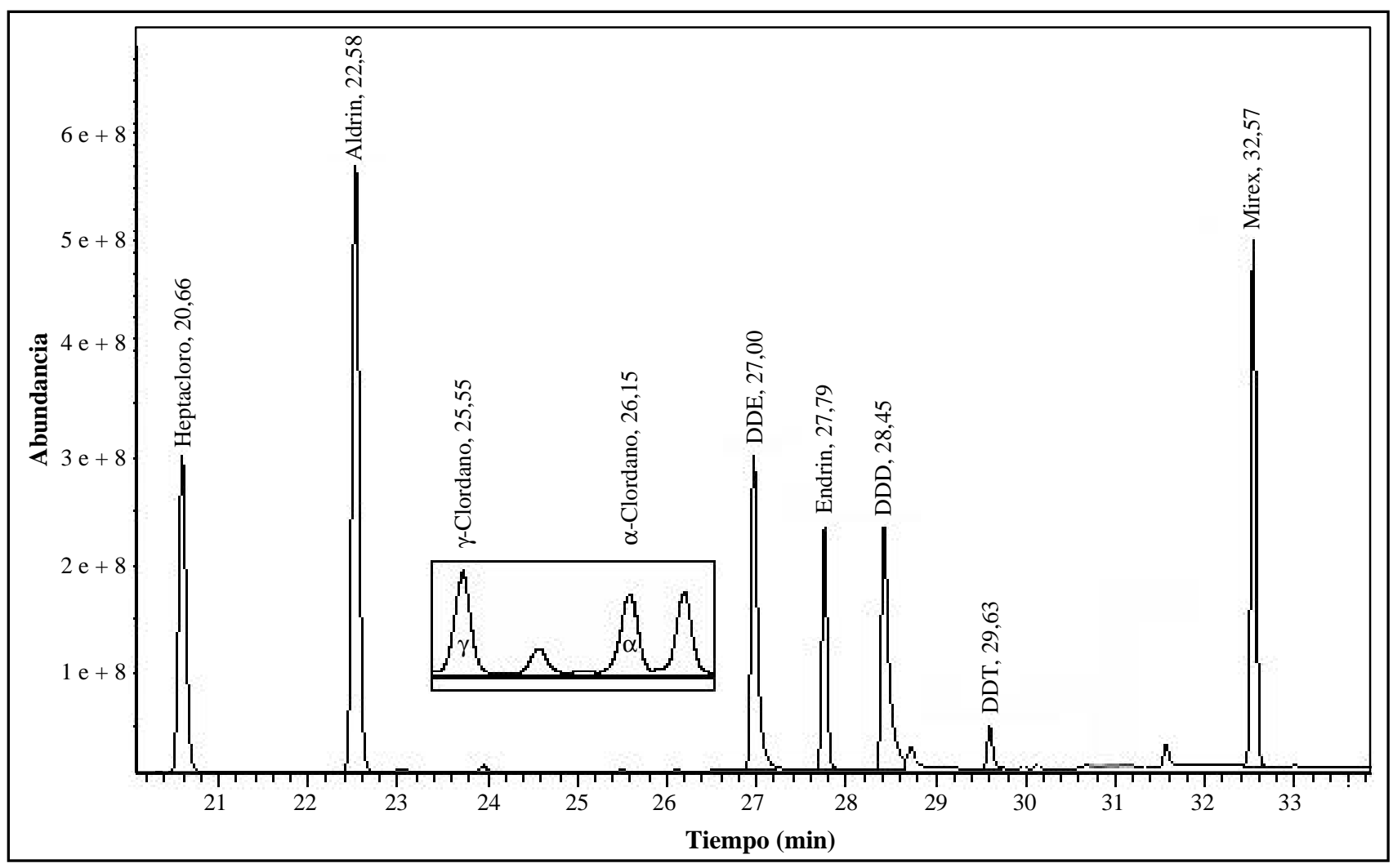

Figura 2. Cromatograma de una mezcla de estándares de pesticidas organoclorados y sus tiempos de retención.

Figure 2. Chromatogram of a mixture of organochlorine pesticide standards and its retention time.

Tabla 1. Recuperación de pesticidas organoclorados añadidos a músculo de carpa común (Cyprinus carpio) usando extracción en fase sólida como método de limpieza, $n=3$; el número entre paréntesis indica la desviación estándar.

Table 1. Recovery of spiked organochlorine pesticides to common carp (Cyprinus carpio) muscle by cleanup with solid-phase extraction method, $n=3$; number in parenthesis is the standard deviation.

\begin{tabular}{lc}
\hline Plaguicida & Recuperación $(\%)$ \\
\hline Heptacloro & $79(6,12)$ \\
Aldrín & $61(2,88)$ \\
Endrín & $91(1,91)$ \\
$\alpha$-Clordano & $59(8,42)$ \\
$\gamma$-Clordano & $56(1,55)$ \\
otros clordanos & $91(5,19)$ \\
Mirex & $57(5,13)$ \\
$p-p$-DDT & $96(8,27)$ \\
$p-p$-DDE & $85(6,99)$ \\
$p$ - $p^{\prime}$-DDD & $80(6,31)$ \\
\hline
\end{tabular}

noreste de Ciudad Victoria y se ubica entre los municipios de Padilla, Güémez, Casas, Abasolo, Jiménez y Soto La Marina en el Estado de Tamaulipas, México.

Los bagres estudiados tenían longitudes de 41 a 43 $\mathrm{cm}$. Su peso varió entre 515 y $685 \mathrm{~g}$ y el peso de su músculo de 152 a 210 g. Los especímenes de carpa fueron los más grandes, con un tamaño que varió de 46 a $69 \mathrm{~cm}$. Su peso total varió de 1430 a 3365 g y el peso de su músculo de 516 a 899 g. Los especímenes de lobina tuvieron un rango en tamaño de 27 a $44 \mathrm{~cm}$, pesaron de 265 a $1115 \mathrm{~g}$ y su músculo varió de 113 a 391 g. Las muestras de tilapia presentaron tamaños entre 30 y $31 \mathrm{~cm}$. Su peso varió entre 555 y 620 g y el peso de su músculo entre 174 y 197 g.

El contenido de humedad es importante porque el reporte de pesticidas se realiza en base húmeda. La humedad en los especímenes de bagre estuvo en el rango de 76,68 a 83,28 \%, en carpa de 80,33 a 83,79\%, en lobina de 79,50 a $81,63 \%$ y en tilapia de 78,60 a 81,64\%. Estos resultados son similares a los obtenidos por otros autores (Schenck et al., 1996), que reportaron valores de 75, 81 y $70 \%$ para pescado azul (bluefish), platija (flounder) y sábalo americano (shad).

Los OCPs son lipofílicos, por lo que se acumulan en la grasa. El contenido de grasa depende de la especie y aún en la misma especie, el contenido graso puede variar debido a cambios estacionales y fisiológicos (Méndez y González, 1997). Los bagres presentaron un contenido de grasa que varió de 1,12 a 5,42 \%, que corresponden a los especímenes con mayor contenido de grasa en este estudio. En las carpas su contenido graso fue de 0,65 a 1,43\%. Las lobinas presentaron valores de grasa entre 0,71 y $2,42 \%$. 
Tabla 2. Concentración en ng/g de pesticidas organoclorados encontrados en músculo de pescado de la Presa Vicente Guerrero, ( $\mathrm{n}=5$ para cada especie). El número entre paréntesis indica la desviación estándar. ND = No detectable, DLD = Debajo del límite de detección.

Table 2. Organochlorine pesticide concentrations (ng/g wet weight) in muscle fish collected in Vicente Guerrero Dam, ( $\mathrm{n}=5$ for each specie). Number in parenthesis indicates the standard deviation. ND $=$ No detectable, DLD $=$ Less than the limit of detection.

\begin{tabular}{|c|c|c|c|c|c|c|c|c|}
\hline \multirow{2}{*}{$\begin{array}{l}\text { Plaguicida } \\
\text { Heptacloro }\end{array}$} & \multicolumn{2}{|c|}{ Bagre } & \multicolumn{2}{|c|}{ Carpa } & \multicolumn{2}{|c|}{ Lobina } & \multicolumn{2}{|c|}{ Tilapia } \\
\hline & ND & & 12,58 & $(7,22)$ & ND & & ND & \\
\hline Aldrín & 0,93 & $(0,61)$ & 3,79 & $(0,13)$ & 0,25 & $(0,56)$ & 0,97 & $(0,55)$ \\
\hline Endrín & 2,40 & $(3,25)$ & 0,80 & $(1,78)$ & 5,21 & $(11,64)$ & 5,16 & $(8,36)$ \\
\hline$\gamma$-Clordano & 1,53 & $(3,41)$ & 11,86 & $(14,43)$ & 7,41 & $(3,81)$ & 10,20 & $(5,92)$ \\
\hline$\alpha$-Clordano & 32,84 & $(46,02)$ & ND & & 1,03 & $(2,30)$ & 7,20 & $(6,97)$ \\
\hline Otros clordanos & 44,66 & $(47,73)$ & 11,86 & $(14,42)$ & 64,22 & $(55,32)$ & 41,40 & $(22,56)$ \\
\hline Mirex & ND & & ND & & DLD & & DLD & \\
\hline$p-p^{\prime}-\mathrm{DDT}$ & 0,93 & $(1,27)$ & 3,44 & $(1,93)$ & 2,79 & $(3,32)$ & 2,54 & $(5,68)$ \\
\hline$p-p$-DDD & 6,34 & $(0,48)$ & 4,11 & $(0,52)$ & 7,08 & $(1,77)$ & 5,91 & $(3,50)$ \\
\hline$p-p$-DDE & 212,31 & $(72,17)$ & 165,98 & $(231,98)$ & 239,90 & $(221,56)$ & 22,75 & $(42,53)$ \\
\hline
\end{tabular}

Los especímenes de tilapia registraron valores de grasa de 1,17 a $1,69 \%$. El contenido de grasa reportado en los especímenes de este estudio es similar al publicado por Schmitt et al. (2005), quienes reportaron valores de 1 a 5 $\%$ de grasa en total y valores de 2 a $7 \%$ de grasa en carpa para especímenes capturados en la Cuenca del Río Grande, localizado en Estados Unidos (Colorado, Nuevo México y Texas) y en la frontera con México. También se considera un contenido de grasa semejante al señalado por Therdteppitak y Yammeng (2003) quienes obtuvieron para tilapia (Oreochromis niloticus) y carpa común (Cyprinus carpio) de Tailandia, 2,55 y 2,19\%, respectivamente, aunque son inferiores a los publicados por Sun et al. (2000), quienes reportaron $11,7 \%$ de grasa en carpa común (Cyprinus carpio) y 5,4\% en tilapia (Tilapia nilotica).

Bajo las condiciones de operación descritas previamente, se obtuvieron los cromatogramas para una serie de estándares y se construyeron las curvas de calibración para cada pesticida, empleando cinco concentraciones para cada analito. Las curvas obtenidas presentaron valores de $\mathrm{R}^{2}$ superiores a 0,990. La Figura 2 muestra un cromatograma para una mezcla de estándares de OCPs y sus tiempos de retención.

La exactitud de un método analítico se determina evaluando la recuperación de los analitos de la matriz de la muestra. La recuperación ideal es de $100 \%$, valores mayores indican interferencias debido a la matriz o errores determinados que deben eliminarse; recuperaciones menores indican pérdidas de analito durante la fase de preparación de la muestra. Cuando la recuperación no es satisfactoria pero es reproducible, cabe la posibilidad de utilizar el método analítico empleando un factor de corrección que compense las pérdidas de analito. En la Tabla 1 se muestran las recuperaciones obtenidas para muestras fortificadas (por triplicado) con una mezcla de pesticidas con concentraciones de $40 \mathrm{ng} / \mathrm{g}$ de músculo de pescado para cada pesticida. El pescado que se usó para fortificar y evaluar las recuperaciones fue carpa.

Las recuperaciones más bajas fueron de 56 y $57 \%$, se observaron para los pesticidas $\gamma$-clordano y mirex, respectivamente. El valor más alto de recuperación fue de $96 \%$ para el DDT. En un trabajo publicado por Sun et al. (2000) también se usó carpa para evaluar la recuperación de pesticidas y una metodología similar de extracción con SPE C18 y florisil, pero usaron niveles de fortificación de $250 \mathrm{ng} / \mathrm{g}$, mientras que en este trabajo el nivel de fortificación fue de $40 \mathrm{ng} / \mathrm{g}$. De los 5 pesticidas que se usaron en común en los 2 trabajos, para el DDT y endrín se obtuvieron recuperaciones similares (91-96\%), para los otros 3 (heptacloro, aldrín y DDE) las recuperaciones en este trabajo fueron menores. Schenck et al. (1996) fortificaron muestras de sábalo con concentraciones de 120 $\mathrm{ng} / \mathrm{g}$, trabajaron con 24 analitos y obtuvieron recuperaciones de 55 a $129 \%$. Sin embargo, la media de recuperación para los 6 analitos en común con este trabajo, es mayor para Schenck et al. (1996) (95\%) que para este estudio $(78 \%)$.

Therdteppitak y Yammeng (2003) usaron el mismo nivel de concentración que en este estudio para fortificar muestras de tilapia, sólo que ellos usaron extracción basada en ultrasonido. Trabajaron con 16 pesticidas pero sólo 5 en común con este estudio. Las recuperaciones de endrín y DDT de este trabajo fueron superiores a las reportadas por Therdteppitak y Yammeng (2003). Sin embargo, para heptacloro, aldrín y DDD ellos obtuvieron mayores recuperaciones, con valores de 94, 89 y $98 \%$, respectivamente. Sun et al., (1996) hicieron un estudio de recuperación de OCPs en pescados con diferente contenido de grasa. Clasificaron a los especímenes en tres grupos, uno con contenido de grasa menor a $10 \%$, otro con valores de 10 a $20 \%$ y el tercer grupo con valores de grasa mayores a $20 \%$. Encontraron que no hubo diferencia significativa en la recuperación de pesticidas de pescados con diferente contenido de grasa.

En el presente trabajo, las especies que presentaron concentraciones más elevadas de OCPs fueron lobina, carpa y bagre. En la Tabla 2 se muestran las concentraciones medias de pesticidas organoclorados encontrados en las muestras de músculo de pescado ( $\mathrm{n}=5$ para cada especie). 
La presencia de $p$ - $p$ '-DDT, el principio activo del DDT comercial, se encontró en el $50 \%$ de las muestras, pero en concentraciones muy bajas. En contraste, residuos de $p$ - $p$ '-DDE, el metabolito más persistente del DDT, estuvo presente en el $90 \%$ de las muestras y en concentraciones mayores. El bagre presentó concentraciones de 122 a $322 \mathrm{ng} / \mathrm{g}$ y la carpa presentó valores de 19 a $562 \mathrm{ng} / \mathrm{g}$. La lobina presentó concentraciones en un intervalo amplio de 5,82 a $584 \mathrm{ng} /$ g. Es pertinente señalar que la lobina que presentó el valor de $584 \mathrm{ng} / \mathrm{g}$ medía solamente $27 \mathrm{~cm}$ y tenía un peso total de $265 \mathrm{~g}$, aunque tenía un tamaño inferior al permitido para su captura, se analizó porque ya estaba muerta cuando se capturó. Mientras que la lobina que presentó el valor de 5,82 medía $43 \mathrm{~cm}$ y pesaba $1115 \mathrm{~g}$. Otros autores también encontraron amplios intervalos para la lobina, por ejemplo valores de 50 a $380 \mathrm{ng} / \mathrm{g}$ (Schmitt et al., 2005).

Los resultados para carpa fueron similares a los de Schmitt et al. (2005) quienes trabajaron en 10 estaciones de muestreo en la Cuenca del Río Grande (USA y frontera con México) y detectaron la presencia de $p$ - $p$ '-DDE en todas las estaciones de muestreo y en 43 de las 47 muestras analizadas (LOD $10 \mathrm{ng} / \mathrm{g}$ ). En carpa encontraron valores de 20 a $520 \mathrm{ng} / \mathrm{g}$ y en bagre valores de 100 y $110 \mathrm{ng} / \mathrm{g}$ en dos estaciones. Aunque los bagres de otra estación mostraron valores de $1430 \mathrm{ng} / \mathrm{g}$.

En los pescados de la Presa Vicente Guerrero se encontraron concentraciones de $p$ - $p$ '-DDD (otro metabolito del DDT) en el $95 \%$ de las muestras. En bagre, los valores fueron de 5,86 a $6,90 \mathrm{ng} / \mathrm{g}$; en carpa las concentraciones fueron de 3,61 a 4,84 ng/g; en lobina los resultados fueron de 5,93 a $10,19 \mathrm{ng} / \mathrm{g}$ y en tilapia de 6,07 a $9,16 \mathrm{ng} / \mathrm{g}$. Estos resultados concuerdan con los obtenidos por Schmitt et al. (2005), ya que ellos encontraron $p$ - $p$ 'DDD en 6 de las 10 estaciones de muestreo y en 23 de las 47 muestras. También en concentraciones uniformemente bajas, menores que $84 \mathrm{ng} / \mathrm{g}$.

Es importante señalar que en el trabajo de Schmitt et al. (2005) el muestreo se llevó a cabo durante los años 1997 y 1998. Una publicación de un muestreo efectuado en el año 2001 en Salton Sea, el lago más grande hecho por el hombre en California, USA, (Sapozhnikova et al., 2004) da valores menores que los encontrados en los pescados de la Presa Vicente Guerrero y del Río Grande para DDE y DDD totales en músculo de tilapia (4,6 a 21,4 y 0,5 a $2,2 \mathrm{ng} / \mathrm{g}$, respectivamente). Los valores mayores encontrados en los pescados de la Presa Vicente Guerrero se pueden atribuir a que en Estados Unidos se prohibió el uso del DDT desde 1972. Sin embargo, el DDT en México aún es utilizado en forma restringida para el control de la malaria (Benítez y Bárcenas, 1996).

Los límites recomendados por la EPA para el total de DDT es de $14 \mathrm{ng} / \mathrm{g}$ para pescado de consumo frecuente (EPA, 2000). Por lo tanto, existe riesgo de cáncer por el consumo de carpa y lobina en forma regular por parte de la población que habita en la región debido a los altos niveles de $p$ - $p$ '-DDE encontrados.

En los pescados de la Presa Vicente Guerrero, el clordano total se detectó en el $85 \%$ de las muestras. Las especies que presentaron la mayor concentración de clordano fueron lobina y bagre (162 y 114 ng/g de músculo, respectivamente), aunque también estuvo presente en todas las muestras de tilapia, sólo que en concentraciones menores $(21,5$ a 78,0 ng/g). En carpa se detectó en concentraciones de 7,9 a 34,4 ng/g. En el estudio de la Cuenca del Río Grande detectaron cis-clordano en 4 de las 10 estaciones y las concentraciones más altas fueron para el bagre, ellos reportaron valores de 17 a $73 \mathrm{ng} / \mathrm{g}$ de cis-clordano en bagre y carpa, resultados similares a los encontrados para tilapia en este trabajo.

Otro pesticida que se encontró en el $70 \%$ de los especímenes estudiados de la Presa Vicente Guerrero fue el aldrín. Todos con valores inferiores a $4 \mathrm{ng} / \mathrm{g}$, donde las concentraciones más altas fueron para carpa, con valores de 3,68 a 3,98 ng/g. Los otros especímenes presentaron en promedio 1,0 ng/g. Therdteppitak y Yammeng (2003) reportaron valores de 0,5 y $0,7 \mathrm{ng} / \mathrm{g}$ de aldrín en muestras de músculo de pescado de las especies Notopterus notopterus y Trichogaster pectoralis de Tailandia.

El endrin se encontró en el $40 \%$ de las muestras, en concentraciones de 1,30 ng/g (en tilapia) a 26,03 ng/g (en lobina). Los valores bajos son similares a los reportados por Sapozhnikova et al. (2004), quienes encontraron valores de 0,7 a 2,2 ng/g en músculo de tilapias del Salton Sea en California, USA.

En la Presa Vicente Guerrero el heptacloro sólo se detectó en carpa, con valores de 14,48 a 18,49 ng/g, ligeramente superiores a los publicados por Sapozhnikova et al. (2004) quienes encontraron valores de 2,5 a 6,7 ng/ g. Como se puede observar en la Tabla 2 , los valores de la suma de clordanos en carpa fueron relativamente bajos comparados con las otras tres especies de pescado, los especímenes de carpa que se analizaron tenían mayor tamaño y peso que los especímenes de las otras especies y existe la posibilidad de que los residuos de clordano detectados provengan de la aplicación de clordano grado técnico, el cual consiste en una mezcla de más de 140 compuestos (Dearth y Hites 1991; Miyazaki et al., 1985) y éste se haya metabolizado a heptacloro (Falandysz et al.,1998).

En los especímenes de la Presa Vicente Guerrero el mirex estuvo por debajo de los límites de detección o no se detectó en las especies estudiadas.

\section{CONCLUSIONES}

Los resultados obtenidos de este estudio, indican que los pesticidas organoclorados aldrín, endrín, mirex, heptacloro, clordanos, $p$ - $p$ '-DDT, $p$ - $p$ '-DDD y $p$ - $p$ '-DDE 
se encuentran presentes en la Presa Vicente Guerrero del estado de Tamaulipas (México) y el $p$ - $p$ '-DDE, un metabolito de la degradación del $p$ - $p$ '-DDT, fue la sustancia que se encontró en mayor concentración en los especímenes analizados.

Existe riesgo de cáncer por el consumo de carpa y lobina, debido a los altos niveles de $p$ - $p$ '-DDE encontrados que sobrepasan los límites recomendados por la EPA, por lo que no se deben consumir estos pescados. Es necesario seguir evaluando su presencia y concentración en este ecosistema, así como establecer alertas para disminuir la frecuencia de consumo de estos pescados.

\section{AGRADECIMIENTOS}

A SEMARNAT-CONACYT por el financiamiento al proyecto SEMARNAT - 2002 - C01- 0462.

\section{BIBLIOGRAFÍA}

Amaral Mendes, J. J. 2002. The endocrine disrupters: a major medical challenge. Food and Chemical Toxicology 40, 781-788.

Brody, J. G.; Rudel, R. A. 2003. Environmental pollutants and breast cancer. Environmental Health Perspectives 111, 1007-1019.

Calle, E. E.; Frumkin, H.; Henley, S. J.; Savitz, D. A.; Thun, M. J. 2002. Organochlorines and breast cancer risk. C A Cancer Journal for Clinicians 52, 301-309.

Benítez, J. A.; Bárcenas, C. 1996. Patrones de uso de los plaguicidas en la zona costera del Golfo de México. In Botello, A. V.; Rojas, J. L.; Benítez, J. A.; ZárateLomelí, D. Golfo de México, Contaminación e impacto ambiental: Diagnóstico y tendencias. EPOMEX Serie Científica 5. Universidad Autónoma de Campeche, México, pp 155-167.

Botello, A. V.; Rueda-Quintana, L.; Diaz-Gonzalez, G.; Toledo, A. 2000. Persistent organochlorine pesticides (POPs) in coastal lagoons of the subtropical Mexican Pacific. Bulletin of Environmental Contamination and Toxicology 64 (3), 390-397.

Carvalho, F. P.; Gonzalez-Farias, F.; Villeneuve, J.-P.; Cattini, C.; Hernandez-Garza, M.; Mee, L. D.; Fowler, S. W. 2002. Distribution, fate and effects of pesticide residues in tropical coastal lagoons of northwestern Mexico. Marine Environment Laboratory, International Atomic Energy Agency, Monaco, Environmental Technology 23 (11), 12571270.

Cruz, S., Lino, C., Silveira, M. I. 2003. Evaluation of organochlorine pesticide residues in human serum from an urban and two rural populations in Portugal. The Science of The Total Environment 317, 23-35.
Dearth, M. A.; Hites, R. A. 1991. Complete Analysis of Technical Chlordane Using Negative Ionization Mass Spectrometry. Environmental Science \& Technology 25, 245-254.

EPA. 2000. Guidance for Assessing Chemical Contaminant Data for Use in Fish Advisories. Volume 2: Risk Assessment and Fish Consumption Limits, Third Edition. U.S. Environmental Protection Agency, Washington, D.C.

Falandysz, J.; Strandberg, B.; Strandberg, L.; Bergqvist, P-A.; Rappe, C. 1998 Concentrations and spatial distribution of chlordanes and some other cyclodiene pesticides in Baltic plankton. Science of the Total Environment 215, 3, 253-258.

Favari, L.; Lopez, E.; Martinez-Tabche, L.; Diaz-Pardo, E. 2002. Effect of Insecticides on Plankton and Fish of Ignacio Ramirez Reservoir (Mexico): A Biochemical and Biomagnification Study. México City, Mex. Ecotoxicology and Environmental Safety 51 (3), 177-186.

Gold-Bouchot, G.; Zapata-Perez, O.; Rodriguez-Fuentes, G.; Ceja-Moreno, V.; del Rio-Garcia, M.; ChanCocom, E. 2006. Biomarkers and pollutants in the Nile Tilapia, Oreochromis niloticus, in four lakes from San Miguel, Chiapas, Mexico. International Journal of Environment and Pollution 26 (1/2/3), 129-141.

Herrera-Portugal, C.; Ochoa, H.; Franco-Sanchez, G.; Yañez., L.; Diaz-Barriga, F. 2005 Environmental pathways of exposure to DDT for children living in a malarious area of Chiapas, Mexico. Environmental Research 99 (2), 158-163.

Hernandez-Romero, A. H.; Tovilla-Hernandez, C.; Malo, E. A.; Bello-Mendoza, R. 2004. Water quality and presence of pesticides in a tropical coastal wetland in southern Mexico. Marine Pollution Bulletin 48 (11-12), 1130-1141.

INE. 2001. Informe final sobre los Avances en la Instrumentación del Plan de Acción Regional de América del Norte sobre Clordano. Comisión para la Cooperación Ambiental de América del Norte.

Iwata, H.; Tanabe, S.; Sakai, N.; Nishimura, A.; Tatsukawa, R. 1994. Geographical distribution of persistent organochlorine in air, water, and sediments from Asia and Oceania and their implications for global redistribution from lower latitudes. Environmental Pollution 85, 15-33.

Lino, C. M., Silveira M. I. N. da. 2006 Evaluation of organochlorine pesticides in serum from students in Coimbra, Portugal: 1997-2001. Environmental Research 102 339-351.

Lewis, M. A.; Scott, G. I.; Bearden, D. W.; Quarles, R. L.; Moore, J. ; Strozier, E. D.; Sivertsen, S. K.; Dias, A. R.; Sanders, M. 2002. Fish tissue quality in nearcoastal areas of the Gulf of Mexico receiving point 
source discharges. Science of the Total Environment 284, (1-3), 249-261.

Lewis, M.; Weber, D.; Stanley, R.; Albrecht, B. 2000. Treated wastewater as a source of sediment contamination in Gulf of Mexico near-coastal areas: a survey. Environmental Toxicology and Chemistry 19, (1), 192-203.

Lewis, M. A.; Weber, D. E.; Stanley, R. S. 1998. Comparative animal and plant toxicities of 10 treated effluents discharged to near-coastal areas of the Gulf of Mexico. Water Environment Research 70 (6), 1108-1117.

Mendez, E.; Gonzalez, R. M. 1997. Seasonal changes in the chemical and lipid composition of fillets of the Southwest Atlantic hake (Merluccius hubbsi). Food Chemistry 59, 213-217.

Miyazaki, T., Yamagishi, T., Matsumoto, M., 1985. Isolation and structure elucidation of some components in technical grade chlordane. Archives of environmental contamination and toxicology $\mathbf{1 4}$, 475-483.

Pazos, P.; Olea-Serrano, M. F.; Zuluaga, A.; Olea, N. 1988. Endocrine disrupting chemicals: xenoestrogens. Médecine Biologie Environnement 26, 1, 41-47.

Sapozhnikova, Y.; Bawardi, O.; Schlenk, D. 2004. Pesticides and PCBs in sediments and fish from the Salton Sea, California, USA. Chemosphere 55, 797-809.

Sun, F.; Lin, F. Y.; Wong, S.S. Li, G. C. 2000. Determination of organochlorine and nitrogen-containing pesticide residues in fish with different fat content. Journal of Food and Drug Analysis 8, 2, 103-111.

Sun, F.; Lin, F. Y.; Wong, S. S. Li, G. C. 2003. The screening of organophosphorous, organochlorine and synthetic pyrethroid pesticides residues in beef fat by tandem solid-phase extraction technique. Journal of Food and Drug Analysis 11, 3, 258-265.
Schenck, F. J.; Wagner, R.; Hennessy, M. K.; Okrasinski, J. L. Jr. 1994. Screening of organochlorine pesticide and polychlorinated biphenyl residues in non fatty seafood products by tandem solid phase extraction clean up. Journal of AOAC International 77, 102106.

Schenck, F. J.; Calderon, L.; Podhorniak, L. V. 1996. Determination of organochlorine pesticide and polichlorinated biphenyl residues in fatty fish by tandem solid - phase extraction cleanup. Journal of AOAC International 79, 1209-1214.

Schmitt, C. J.; Hinck, J. E.; Blazer, V. S.; Denslow, N. D.; Dethloff, G. M.; Bartish, T. M.; Coyle, J.J.; Tillitt, D. E. 2005. Environmental contaminants and biomarker responses in fish from the Rio Grande and its U.S. tributaries: Spatial and temporal trends. Science of the Total Environment 350, 161-193.

Therdteppitak, A.; Yammeng, K. 2003. Determination of organochlorine pesticides in commercial fish by gas chromatography with electron capture detector and confirmation by gas chromatography- mass spectrometry. Science Asia 29, 127-134.

UNEP. 2001. Stockholm Convention on POPs, Text and Annexes, Interim Secretariat for the Stockholm Convention on Persistent Organic Pollutant, UNEP Chemicals, Geneva, Switzerland.

UNEP. 2007. Programa de las Naciones Unidas para el Medio Ambiente. Convenio de Estocolmo sobre contaminantes orgánicos persistentes. Comité de Examen de los Contaminantes Orgánicos Persistentes. Evaluación de la bioacumulación. Tercera reunión. Ginebra, 19 al 23 de noviembre.

Vinggaard, A. M.; Breinholt, V.; Larsen, J. C.; 1999. Screening of selected pesticides for oestrogen receptor activation in vitro. Food Additives \& Contaminants 16, 12, $533-542$. 